Review began 12/10/2021 Review ended 01/09/2022 Published 01/16/2022

๑) Copyright 2022

Umeh et al. This is an open access article distributed under the terms of the Creative Commons Attribution License CC-BY 4.0. which permits unrestricted use, distribution, and reproduction in any medium, provided the original author and source are credited.

\section{A Multicenter Retrospective Analysis on the Etiology of Bradycardia in COVID-19 Patients}

Chukwuemeka Umeh ${ }^{1}$, Curren Giberson ${ }^{1}$, Sabina Kumar ${ }^{1}$, Mahendra Aseri ${ }^{2}$, Pranav Barve ${ }^{1}$

1. Internal Medicine, Hemet Global Medical Center, Hemet, USA 2 2. Data Engineering and Business Intelligence, Hemet Global Medical Center, Hemet, USA

Corresponding author: Chukwuemeka Umeh, emmyumeh@yahoo.com

\section{Abstract \\ Introduction}

Bradycardia has been reported in the setting of SARS-CoV2 (COVID-19) and appears to be an important cardiac manifestation with an association of mortality. However, the etiology of bradycardia in COVID-19 remains unclear. Therefore, this study aims to retrospectively investigate the potential causes of bradycardia in COVID-19 patients.

\section{Method}

The multicenter retrospective analysis consisted of 1,116 COVID-19 positive patients from March 2020 to March 2021. Bradycardia and severe bradycardia were defined as a sustained heart rate of $<60$ BPM and $<50$ $\mathrm{BPM}$, respectively, on two separate occasions, a minimum of four hours apart during the hospitalization. End-of-life bradycardia was excluded from the study. Data were retrieved using a structured query language (SQL) program through the EMR, and data were analyzed using IBM SPSS 27.0 (IBM Corp., Armonk, NY). Logistic regression was used to study the bradycardic event and its association with remdesivir, betablockers, or steroids use during the patient's hospital stay.

\section{Result}

In the multivariate analysis, bradycardia was significantly associated with length of hospital stay $(\mathrm{p}<0.001)$, mortality ( $\mathrm{p}=0.022)$, ventilator use $(\mathrm{p}=0.001)$, and steroid use $(\mathrm{p}=0.001)$. However, there was no significant association between bradycardia and remdesivir use $(\mathrm{p}=0.066)$ or beta-blocker use $(\mathrm{p}=0.789)$.

\section{Conclusion}

Our study showed that steroid use was protective against developing bradycardia in COVID-19 patients. Furthermore, remdesivir and the use of beta-blockers were not associated with bradycardia in COVID-19 patients. However, bradycardia was associated with both increased mortality and length of stay in the hospital. Therefore, future studies should focus on the mechanism of bradycardia in COVID-19 patients and the effect of bradycardia on patient outcomes.

Categories: Cardiology, Internal Medicine, Infectious Disease

Keywords: mortality, beta-blocker, steroid, remdesivir, bradycardia, sars-cov2, covid-19

\section{Introduction}

Coronavirus disease 2019 (COVID-19), caused by severe acute respiratory syndrome coronavirus 2 (SARSCoV-2), originated in Wuhan, China, in December 2019 and has since spread to nearly every country on the planet. As of November 2021, there have been 258 million cases and 5.16 million deaths worldwide. In the United States alone, there have been 47.9 million confirmed cases and 770,890 deaths [1].

SARS-CoV-2, like many viruses, must bind to a cell surface protein to facilitate entry into host cells. It utilizes the receptor-binding domain (RBD) of its spike (S) protein to bind to human angiotensin-converting enzyme-2 (ACE2) [2], a protein present on nearly all human tissues but particularly abundant in the heart, kidneys, gastrointestinal tract, and respiratory system [3]. There is high expression of the ACE2 gene in nasal epithelial cells, possibly contributing to the high infectivity and rapid spread of SARS-CoV-2 [4]. Expression of the RAS is a complex and highly regulated process that pathogens can manipulate. Some viruses, such as SARS-CoV-2, induce transcription of type 1 interferon (IFN-a), a pro-inflammatory cytokine produced by human respiratory epithelial cells, indirectly enhancing expression of ACE2 and facilitating viral entry into host cells [5].

The symptoms of SARS-CoV-2 infection highly correlate with the organs that have the highest ACE2 expression. Infection of the respiratory and gastrointestinal epithelium produces common symptoms such as dyspnea, cough, and diarrhea. However, a less-publicized implication of COVID-19 is cardiac damage. 
Patients without cardiovascular comorbidities who become infected with SARS-CoV-2 have an increased incidence of arrhythmias, cardiomyopathies, acute coronary syndromes, coagulopathies, and myocarditis [6], while patients with preexisting cardiovascular disease have increased mortality [7]. While the exact pathophysiology is unclear, SARS-CoV-2 arrhythmias are thought to arise from electrolyte abnormalities, acidosis, hypoxemia, and inflammation resulting from the massive release of cytokines, also known as a cytokine storm [8].

One proposed mechanism for the bradyarrhythmia seen in COVID-19 infection is the correlation between the cytokine storm produced by severe SARS-CoV-2 infection and elevated interleukin-6 (IL-6) [9]. IL-6 has been shown to directly affect the sinoatrial node, causing increased vagal tone and decreased heart rate [6]. In addition, medications used in treating COVID-19 are also a possible cause of bradycardia in COVID-19 patients $[10,11]$. The rate of bradyarrhythmia among all COVID-19 patients in Wuhan, China, has been reported as high as $16.7 \%$ [12], but the incidence increases significantly among hospitalized patients with severe COVID-19 $[9,13]$. The most common bradyarrhythmias among hospitalized COVID-19 patients were sinus bradycardia and first-degree heart block [14]. While tachyarrhythmias are more common in COVID-19 patients, bradyarrhythmia has been associated with higher mortality and more severe disease [15]. Therefore, this study aims to retrospectively investigate the potential causes of bradycardia in COVID-19 patients.

\section{Materials And Methods}

We performed a multicenter retrospective analysis, which included two Southern California hospitals, on patients with a COVID-19 diagnosis verified by PCR between March 2020 and March 2021. A total of 1,116 patients were identified. Relevant deidentified patient data were extracted using a structured query language (SQL) program from the electronic medical record, which included: age, gender, race, comorbidities, laboratory results on admission, date of admission, date of discharge, medications they received while on admission, heart rate, and disposition at discharge. Bradycardia and severe bradycardia were defined as a sustained heart rate $<60$ beats per minute and $<50$ beats per minute, respectively, on two separate occasions, a minimum of four hours apart during the hospitalization [16]. End-of-life bradycardia was excluded from the study.

We performed a univariate analysis of the independent variables, including patients' age, gender, ethnicity, marital status, comorbidities, the medication patients received while in the hospital, and laboratory results, using means and percentages. Furthermore, we performed a bivariate analysis of the relationship between bradycardia and different study variables using chi-square and t-test, with a P-value of 0.05 considered significant. Finally, we performed a backward selection logistic regression to study the relationship between bradycardia, remdesivir, and steroid use. The effect was expressed as an odds ratio with a 95\% confidence interval. Statistical analysis was done using IBM SPSS version 27. The WIRB-Copernicus Group (WCG) institutional review board (IRB) approved the study, and the study's IRB approval number is 13410516.

\section{Results}

We had 1,116 patients in the study, with a mean age of 66 years and a range of 19 to 101 years. The mean hospital length of stay was nine days and ranged from 0 to 64 days (Table 1). Forty-nine percent of the patients were female, $82 \%$ were white, and $26 \%$ expired. Forty-five percent of the patients received remdesivir, and $66 \%$ received steroids (dexamethasone or methylprednisolone; Table 1 ).

\begin{tabular}{|c|c|c|}
\hline & Mean & Standard deviation \\
\hline Age & 65.52 & 17.512 \\
\hline Body mass index & 30.786 & 8.9036 \\
\hline \multirow[t]{2}{*}{ Length of hospital stay } & 9.03 & 8.199 \\
\hline & Frequency & Percent \\
\hline \multicolumn{3}{|l|}{ Gender } \\
\hline Female & 541 & $48.5 \%$ \\
\hline Male & 575 & $51.5 \%$ \\
\hline \multicolumn{3}{|l|}{ Race } \\
\hline White & 900 & $81.6 \%$ \\
\hline Black & 80 & $7.2 \%$ \\
\hline Others & 125 & $11.2 \%$ \\
\hline
\end{tabular}




\section{Cureus}

Expired

$\begin{array}{ll}\text { No } & 821\end{array}$

$73.6 \%$

Yes

295

$26.4 \%$

Ventilator use

No

909

81.5

Yes

207

$18.5 \%$

ICU admission

No

878

Yes

238

$21.3 \%$

Remdesivir

No

609

$54.6 \%$

Yes

507

$45.4 \%$

Steroid use (dexamethasone or methylprednisolone)

No

Yes

735

$65.9 \%$

Diabetes

No

Yes

Hypertension

No

Chronic kidney disease

No

Yes

889

227

Acute kidney injury

No

Yes

822

294

Coronary artery disease

No

Yes

910

206

Congestive heart failure

No

Yes

Chronic obstructive pulmonary disease

No

Yes

157

Beta-blocker use

No

Yes

Pulse $<50$ beats per minute
$73.7 \%$

$26.3 \%$

$81.5 \%$

$18.5 \%$

$82.6 \%$

$17.4 \%$

$14.1 \%$

$69 \%$

$31 \%$ 


\section{Cureus}

No

Yes

Pulse $<60$ beats per minute

No

Yes

TABLE 1: Descriptive statistics of all patients in the study
93.1

6.9

$66.3 \%$

$33.7 \%$

In the bivariate analysis, mortality $(\mathrm{p}=0.001)$, ventilator use $(\mathrm{p}<0.001)$, intensive care unit admission $(\mathrm{p}<0.001)$, diabetes $(\mathrm{p}=0.002)$, hypertension $(\mathrm{p}=0.02)$, remdesivir use $(\mathrm{p}<0.001)$, steroid use $(\mathrm{p}<0.001)$, and calcium channel blocker use ( $\mathrm{p}=0.003$ ) were significantly associated with bradycardia (Table 2 ).

\begin{tabular}{|c|c|c|c|}
\hline \multirow{2}{*}{ Variable } & \multicolumn{2}{|c|}{ Bradycardia } & \multirow{2}{*}{ P-value } \\
\hline & No & Yes & \\
\hline \multicolumn{4}{|l|}{ Gender } \\
\hline Male & $64.5 \%$ & $35.5 \%$ & 0.193 \\
\hline Female & $68.2 \%$ & $31.8 \%$ & \\
\hline \multicolumn{4}{|l|}{ Race } \\
\hline White & $66.6 \%$ & $33.4 \%$ & 0.413 \\
\hline Black & $70.0 \%$ & $30.0 \%$ & \\
\hline Others & $61.6 \%$ & $38.4 \%$ & \\
\hline \multicolumn{4}{|l|}{ Expired } \\
\hline Yes & $58.3 \%$ & $41.7 \%$ & 0.001 \\
\hline No & $69.2 \%$ & $30.8 \%$ & \\
\hline \multicolumn{4}{|c|}{ Ventilator use } \\
\hline Yes & $44.0 \%$ & $56.0 \%$ & $<0.001$ \\
\hline No & $71.4 \%$ & $28.6 \%$ & \\
\hline \multicolumn{4}{|c|}{ Intensive care unit admission } \\
\hline Yes & $49.6 \%$ & $50.4 \%$ & $<0.001$ \\
\hline No & $70.8 \%$ & $29.2 \%$ & \\
\hline \multicolumn{4}{|l|}{ Diabetes } \\
\hline Yes & $61.5 \%$ & $38.5 \%$ & 0.002 \\
\hline No & $70.2 \%$ & $29.8 \%$ & \\
\hline \multicolumn{4}{|c|}{ Hypertension } \\
\hline Yes & $63.6 \%$ & $36.4 \%$ & 0.02 \\
\hline No & $70.4 \%$ & $29.6 \%$ & \\
\hline \multicolumn{4}{|c|}{ Chronic kidney disease } \\
\hline Yes & $69.6 \%$ & $30.4 \%$ & 0.239 \\
\hline No & $65.5 \%$ & $34.5 \%$ & \\
\hline \multicolumn{4}{|c|}{ Acute kidney injury } \\
\hline Yes & $61.9 \%$ & $38.1 \%$ & 0.063 \\
\hline
\end{tabular}




\section{Cureus}

No

Congestive heart failure

Yes

No

Chronic obstructive pulmonary disease

Yes

No

Coronary artery disease

Yes

No

Remdesivir use

Yes

No

Steroid use

Yes

No

Beta-blocker use

Yes

No

Calcium channel blockers use

Yes

No

Statin

Yes

No
$67.9 \%$

$32.1 \%$

$64.9 \%$

$35.1 \%$

0.659

$66.6 \%$

$33.4 \%$

$29.3 \%$

$34.4 \%$

$65.6 \%$

$65.5 \%$

$34.5 \%$

0.795

$66.5 \%$

$33.5 \%$

$43.0 \%$

$<0.001$

$74.1 \%$

$25.9 \%$

$59.2 \%$

$80.1 \%$

$65.0 \%$

$66.9 \%$

$58.9 \%$

$68.7 \%$

$65.2 \%$

$67.2 \%$
$35.0 \%$

0.544

$33.1 \%$

$40.8 \%$

$<0.001$

$19.9 \%$

$31.3 \%$

$34.8 \%$

0.479

$32.8 \%$

TABLE 2: Bivariate analysis of the relationship between categorical variables and bradycardia

In the backward selection logistic regression multivariate analysis, bradycardia was significantly associated with length of hospital stay ( $\mathrm{p}<0.001)$, mortality $(\mathrm{p}=0.022)$, ventilator use $(\mathrm{p}=0.001)$, and steroid use $(p=0.001)$. However, there was no significant association between bradycardia and remdesivir use $(p=0.066)$ or beta-blocker use $(\mathrm{p}=0.789)$ (Table 3). 


\section{Cureus}

\begin{tabular}{|c|c|c|c|c|c|c|c|c|}
\hline & \multirow{2}{*}{ B } & \multirow{2}{*}{ S.E. } & \multirow{2}{*}{ Wald } & \multirow{2}{*}{ df } & \multirow{2}{*}{ P-value } & \multirow{2}{*}{ Odds ratio } & \multicolumn{2}{|c|}{$95 \% \mathrm{Cl}$ for odds ratio } \\
\hline & & & & & & & Lower & Upper \\
\hline Length of hospital stay & 0.069 & 0.011 & 42.748 & 1 & 0.000 & 1.071 & 1.049 & 1.094 \\
\hline Age & 0.008 & 0.004 & 3.158 & 1 & 0.076 & 1.008 & 0.999 & 1.016 \\
\hline Expired & 0.470 & 0.205 & 5.239 & 1 & 0.022 & 1.600 & 1.070 & 2.394 \\
\hline Ventilator use & -0.755 & 0.233 & 10.480 & 1 & 0.001 & 0.470 & 0.298 & 0.742 \\
\hline Steroid use & -0.582 & 0.168 & 12.018 & 1 & 0.001 & 0.559 & 0.402 & 0.776 \\
\hline Diabetes & -0.264 & 0.139 & 3.605 & 1 & 0.058 & 0.768 & 0.585 & 1.009 \\
\hline Beta-blocker use & 0.041 & 0.154 & 0.072 & 1 & 0.789 & 1.042 & 0.770 & 1.410 \\
\hline Remdesivir use & -0.278 & 0.151 & 3.378 & 1 & 0.066 & 0.758 & 0.563 & 1.019 \\
\hline
\end{tabular}

TABLE 3: Multivariate analysis of the relationship between bradycardia and different variables

\section{Discussion}

Our analysis showed that 34\% of COVID-19 patients had bradycardia, while $7 \%$ had severe bradycardia during their hospital stay. The cause of bradycardia in our study is unclear as bradycardia was not associated with atrioventricular nodal blocking agents such as beta-blockers. Prior studies have also noted that bradycardia in COVID-19 patients was not associated with hypoxia, myocardial ischemia, or medications that induce bradycardia [16,17]. Therefore, it is possible that the bradycardia in our study was caused by a direct pathogenic effect of COVID-19 on the myocardium or conduction system or that the COVID-19 infection worsened preexisting myocardial or conduction system conditions in the patients [18]. In addition, bradycardia was associated with an increased length of hospital stay and mortality in our study. The association of bradycardia with increased mortality has been reported in previous studies; however, the mechanism remains unclear $[16,17,19]$. One possible explanation is that the inflammatory cytokine storm in COVID-19 both causes bradycardia by its effect on pacemaker cells and also causes increased mortality $[16,17,19]$.

Our study showed that the use of dexamethasone or methylprednisolone was protective against bradycardia, which means that patients on these steroids were significantly less likely to have bradycardia. Contrary to our finding, steroids have been reported to cause bradycardia in non-COVID-19 patients, especially after using high or pulse-dose steroids [20-22]. We had expected that steroid use would result in bradycardia in COVID-19 patients, but the reverse was the case. The mechanism through which steroids provide a protective effect against bradycardia is unclear. A possible explanation is that bradycardia in COVID-19 is caused by damage to the myocardium and conduction system from inflammatory system activation and cytokine storm [19]. In this scenario, steroids inhibit the severe inflammatory process of COVID-19 and reduce bradycardia in COVID-19 patients [23]. Another possible explanation is that corticosteroids act on beta-adrenergic receptors in the heart, leading to positive inotropic and chronotropic effects [23,24]. Thus, the increased heart rate from steroid use may counteract the bradycardia caused by COVID-19 infection.

Furthermore, contrary to prior studies, our study did not show any association between remdesivir use and bradycardia. Some authors have reported bradycardia in COVID-19 patients on remdesivir, which happens through an unclear mechanism [25-28]. It has been postulated that remdesivir may cause bradycardia by slowing the sinoatrial node's automaticity through its active metabolite, a nucleotide triphosphate derivative with a similarity to adenosine triphosphate (ATP) [26,27]. However, no randomized controlled trial has reported increased bradycardia in patients on remdesivir. Thus, the bradycardia seen in observational studies and case reports might have been due to confounders.

The strengths of our study include a multicenter study with a large sample size ( $>1000$ patients) that relied on clinical, laboratory, and outcome data collection. Additionally, we adjusted for possible confounders that would have affected bradycardia, such as the use of beta-blockers. However, our study was limited by its retrospective cohort design. Our study initially included all patients with a documented COVID-19 infection from two southern California hospitals. In addition, the data collection was dependent on the results entered into the electronic medical record, which could cause some discrepancies. Despite our careful analysis, it is not possible to fully account for all potential confounders that might affect the outcome of our study.

\section{Conclusions}


Our study demonstrated that dexamethasone or methylprednisolone use was protective against developing bradycardia in COVID-19 patients. Furthermore, remdesivir and the use of beta-blockers were not associated with bradycardia in COVID-19 patients. However, bradycardia was associated with increased mortality and an increased length of hospital stay. Therefore, in the future, studies should focus on the mechanism of bradycardia in COVID-19 patients and the effect of bradycardia on patient outcomes.

\section{Additional Information \\ Disclosures}

Human subjects: Consent was obtained or waived by all participants in this study. WIRB-Copernicus Group (WCG) issued approval 13410516. Animal subjects: All authors have confirmed that this study did not involve animal subjects or tissue. Conflicts of interest: In compliance with the ICMJE uniform disclosure form, all authors declare the following: Payment/services info: All authors have declared that no financial support was received from any organization for the submitted work. Financial relationships: All authors have declared that they have no financial relationships at present or within the previous three years with any organizations that might have an interest in the submitted work. Other relationships: All authors have declared that there are no other relationships or activities that could appear to have influenced the submitted work.

\section{References}

1. COVID data tracker. (2021). Accessed: November 24, 2021: https://covid.cdc.gov/covid-data-tracker/.

2. Hoffmann M, Kleine-Weber H, Schroeder S, et al.: SARS-CoV-2 cell entry depends on ACE2 and TMPRSS2 and is blocked by a clinically proven protease inhibitor. Cell. 2020, 181:271-280.e8. 10.1016/j.cell.2020.02.052

3. Gkogkou E, Barnasas G, Vougas K, Trougakos IP: Expression profiling meta-analysis of ACE2 and TMPRSS2, the putative anti-inflammatory receptor and priming protease of SARS-CoV-2 in human cells, and identification of putative modulators. Redox Biol. 2020, 36:101615. 10.1016/i.redox.2020.101615

4. Sungnak W, Huang N, Bécavin C, et al.: SARS-CoV-2 entry factors are highly expressed in nasal epithelial cells together with innate immune genes. Nat Med. 2020, 26:681-7. 10.1038/s41591-020-0868-6

5. Ziegler CG, Allon SJ, Nyquist SK, et al.: SARS-CoV-2 receptor ACE2 is an interferon-stimulated gene in human airway epithelial cells and is detected in specific cell subsets across tissues. Cell. 2020, 181:10161035.e19. 10.1016/j.cell.2020.04.035

6. Douedi S, Mararenko A, Alshami A, et al.: COVID-19 induced bradyarrhythmia and relative bradycardia: an overview. J Arrhythm. 2021, 37:888-92. 10.1002/joa3.12578

7. Guo T, Fan Y, Chen M, et al.: Cardiovascular implications of fatal outcomes of patients with coronavirus disease 2019 (COVID-19). JAMA Cardiol. 2020, 5:811-8. 10.1001/jamacardio.2020.1017

8. Thakkar AN, Tea I, Al-Mallah MH: Cardiovascular implications of COVID-19 infections. Methodist Debakey Cardiovasc J. 2020, 16:146-54. 10.14797/mdcj-16-2-146

9. Capoferri G, Osthoff M, Egli A, Stoeckle M, Bassetti S: Relative bradycardia in patients with COVID-19. Clin Microbiol Infect. 2021, 27:295-6. 10.1016/i.cmi.2020.08.013

10. Gupta AK, Parker BM, Priyadarshi V, Parker J: Cardiac adverse events with remdesivir in COVID-19 infection. Cureus. 2020, 12:e11132. 10.7759/cureus.11132

11. Khandelwal K, Madathala RR, Chennaiahgari N, Yousuffuddin M: Steroid-induced sinus bradycardia. Cureus. 2021, 13:e15065. 10.7759/cureus.15065

12. Wang D, Hu B, Hu C, et al.: Clinical characteristics of 138 hospitalized patients with 2019 novel coronavirusinfected pneumonia in Wuhan, China. JAMA. 2020, 323:1061-9. 10.1001/jama.2020.1585

13. Hu L, Gong L, Jiang Z, Wang Q, Zou Y, Zhu L: Clinical analysis of sinus bradycardia in patients with severe COVID-19 pneumonia. Crit Care. 2020, 24:257. 10.1186/s13054-020-02933-3

14. Gopinathannair R, Merchant FM, Lakkireddy DR, et al.: COVID-19 and cardiac arrhythmias: a global perspective on arrhythmia characteristics and management strategies. J Interv Card Electrophysiol. 2020, 59:329-36. 10.1007/s10840-020-00789-9

15. Amaratunga EA, Corwin DS, Moran L, Snyder R: Bradycardia in patients with COVID- 19: a calm before the storm?. Cureus. 2020, 12:e8599. 10.7759/cureus.8599

16. Kumar S, Arcuri C, Chaudhuri S, Gupta R, Aseri M, Barve P, Shah S: A novel study on SARS-COV-2 virus associated bradycardia as a predictor of mortality-retrospective multicenter analysis. Clin Cardiol. 2021, 44:857-62. 10.1002/clc.23622

17. Chalkias A, Pantazopoulos I, Papagiannakis N, et al.: Sinus bradycardia is associated with poor outcome in critically ill patients with COVID-19 due to the B.1.1.7 Lineage. Toxicol Rep. 2021, 8:1394-8. 10.1016/j.toxrep.2021.07.004

18. Kochi AN, Tagliari AP, Forleo GB, Fassini GM, Tondo C: Cardiac and arrhythmic complications in patients with COVID-19. J Cardiovasc Electrophysiol. 2020, 31:1003-8. 10.1111/jce.14479

19. Chinitz JS, Goyal R, Harding M, et al.: Bradyarrhythmias in patients with COVID-19: marker of poor prognosis?. Pacing Clin Electrophysiol. 2020, 43:1199-204. 10.1111/pace.14042

20. Taylor MR, Gaco D: Symptomatic sinus bradycardia after a treatment course of high-dose oral prednisone . J Emerg Med. 2013, 45:e55-8. 10.1016/j.jemermed.2013.04.020

21. Akikusa JD, Feldman BM, Gross GJ, Silverman ED, Schneider R: Sinus bradycardia after intravenous pulse methylprednisolone. Pediatrics. 2007, 119:e778-82. 10.1542/peds.2006-0029

22. Stroeder J, Evans C, Mansell H: Corticosteroid-induced bradycardia: case report and review of the literature . Can Pharm J (Ott). 2015, 148:235-40. 10.1177/1715163515597451

23. Sapolsky RM, Romero LM, Munck AU: How do glucocorticoids influence stress responses? Integrating permissive, suppressive, stimulatory, and preparative actions. Endocr Rev. 2000, 21:55-89. 


\section{Cureus}

10.1210/edrv.21.1.0389

24. Davies AO, Lefkowitz RJ: Regulation of beta-adrenergic receptors by steroid hormones . Annu Rev Physiol. 1984, 46:119-30. 10.1146/annurev.ph.46.030184.001003

25. Pallotto C, Suardi LR, Gabbuti A, Esperti S, Mecocci L, Blanc P: Potential remdesivir-related transient bradycardia in patients with coronavirus disease 2019 (COVID-19). J Med Virol. 2021, 93:2631-4. 10.1002/jmv.26898

26. Touafchia A, Bagheri H, Carrié D, Durrieu G, Sommet A, Chouchana L, Montastruc F: Serious bradycardia and remdesivir for coronavirus 2019 (COVID-19): a new safety concerns. Clin Microbiol Infect. 2021, 10.1016/j.cmi.2021.02.013

27. Barkas F, Styla CP, Bechlioulis A, Milionis H, Liberopoulos E: Sinus bradycardia associated with remdesivir treatment in COVID-19: a case report and literature review. J Cardiovasc Dev Dis. 2021, 8:10.3390/jcdd8020018

28. Gubitosa JC, Kakar P, Gerula C, et al.: Marked sinus bradycardia associated with remdesivir in COVID-19: a case and literature review. JACC Case Rep. 2020, 2:2260-4. 10.1016/j.jaccas.2020.08.025 